\title{
Asymptomatic lymphangioma involving the spleen and mediastinum in adults
}

\section{Afshin Mohammadi ${ }^{1}$, Mohammad Ghasemi-rad ${ }^{2}$, Fariba Abassi ${ }^{3}$}

${ }^{1}$ Department of Radiology, ${ }^{2}$ Student Research Center, ${ }^{3}$ Department of Pathology, Urmia University of Medical Sciences, Urmia, West-Azerbaijan, Iran

\section{Abstract}

Developmental malformation of the lymphatic system and cystic proliferation of lymphatic vessels causes a very rare condition known as cystic lymphangioma. The malformation may involve the spleen alone or be a part of multiorgan involvement. We describe a case of cystic splenic cystic lymphangioma accompanied with mediastineal lymphangioma in a middle age woman with the emphasis on the rarity of the case in adults and the difficulties of the differential diagnosis.

Keywords: mediastinum, spleen, lymphangioma, ultrasonography

\section{Introduction}

Cystic lymphangiomas are benign vascular tumors originating from the lymphatic channels found in different organs. They are most commonly discovered in children and are located in the head and neck region [1]. Splenic cystic lymphangioma is an extremely rare tumour [2,3], and simultaneous splenic and mediastineal involvement is exceptional. We present an asymptomatic case of a 36-year-old woman with a simultaneous cystic lymphangioma situated in the spleen and mediastineoum discovered incidentally during routine ultrasound examination in pregnancy. This case is presented to emphasise the differential diagnosis of cystic lesions involving multiple organ systems.

\section{Case report}

A 36-year-old female patient was admitted with history of palpable spleen $3 \mathrm{~cm}$ under costal margin acciden-

Received 21.02.2013 Accepted 26.03.2013

Med Ultrason

2013, Vol. 15, No 2, 154-156

Corresponding author: Afshin Mohammadi

MD, Department of Radiology

Urmia University of Medical Sciences

Urmia, West-Azerbaijan, Iran

Tel: +98441 3455810, Fax: +984412353561

Email: mohamadi_afshin@yahoo.com tally found 1 year prior to current presentation. She had no significant medical past history, fever, weight loss, or abdominal pain. Physical examination revealed a palpable mass without tenderness or rebound tenderness in the left upper quadrant. All biochemical and hematologic tests (complete blood count, erythrocyte sedimentation rate, C-reactive protein, blood urea nitrogen, creatinin, alanine amino transferase, aspartate amino transferase, and lactate dehydrogenase) were within normal ranges.

The patient underwent abdominal ultrasonography that revealed an enlarged spleen with a bipolar diameter of $190 \mathrm{~cm}$. The spleen contained many varying size cystic lesions ( 1 up to $3.5 \mathrm{~cm}$ in diameter) without septation or solid mural nodule (fig 1). These ultrasonographic findings were the same as the findings reported 12 months before the current examination. The chest X-Ray examination showed the widening of mediastinum with normal bronchovascular marking. Thoracho-abdominal contrast enhanced computed tomographic (CT) scanning demonstrated the enlarged and cystic spleen together with a large low attenuating mass encasing the great vessels of mediastinum and esophagus. It extended from the carina to the retero-crural area of the diaphragm (fig 2, fig 3).

This type of involvement suggested a lymphoproliferative or parasitic process (hydatidosis). To clarify whether these lesions were related to either one, the patient underwent a surgical spleenectomy. Abdominal operation 
showed a large spleen consisting of many cystic lesions (fig 4). The abdominal operation showed the same cystic lesion extending to the mediastinum from the abdominal portion of the esophagus through the retrocrural portion of the diaphragm. Histopathology examination revealed multiple proteinaceous fluid filled vascular spaces lined by endothelial cells associated with fibrous trabeculae; the spaces were lined by flat endothelial cells (CD 1 positive) and were filled with proteinaceous fluid (fig 5).

The patient remains in good general condition 6 months after surgery and without complications.

\section{Discussion}

Cystic lymphangioma is a malformed development of the lymphatic channels. The vast majority of lymphangiomas occur under the age of 2 years with no predisposition with either sex [1]. The neck (75\%) and axillary regions (20\%) are the most common sites involved by lymphangioma, but infrequently it is been reported in the retroperitoneum, mediastinum, mesentery, omentum, colon, pelvis, groin, bone, skin, scrotum and spleen [4]. Less than $1 \%$ of lymphangiomas are localized intra-abdominally.

The pathogenesis of the lymphangiomas is not well known. It is believed that abnormal congenital development, bleeding or inflammation of the lymphatic system may cause progressive dilatation of lymphatic vessels [5]. This is because of insufficient drainage due to atresia or inadequate efferent channels [5].

Lymphangiomas are usually asymptomatic and they are discovered incidentally during unrelated abdominal ultrasonography or computed tomography [6]. They can be seen as single or multiple round cystic, multiloculated or uniloculated structures. Most of them are asymptomatic and a sudden enlargement of lymphangioma due to internal bleeding, infections, or by compression to adjacent organs is the most common cause of symptomatic lymphangioma [7].

The most common clinical manifestations of splenic lymphangioma are left upper quadrant pain, frequently accompanied by fever, nausea and vomiting. Like our case report, splenic lymphangioma can be asymptomatic and incidentally diagnosed. Our case is unique because lymphangioma was found both in her spleen and mediastinum. No report is available on lymphangioma involving these two organs simultaneously in an asymptomatic patient.

Lymphangioma involving more than two organs is rarely reported in literature $[8,9]$. Chung et al reported lymphangioma involving the spleen and retroperitoneum in an asymptomatic adult [8]. Kim et al reported the only

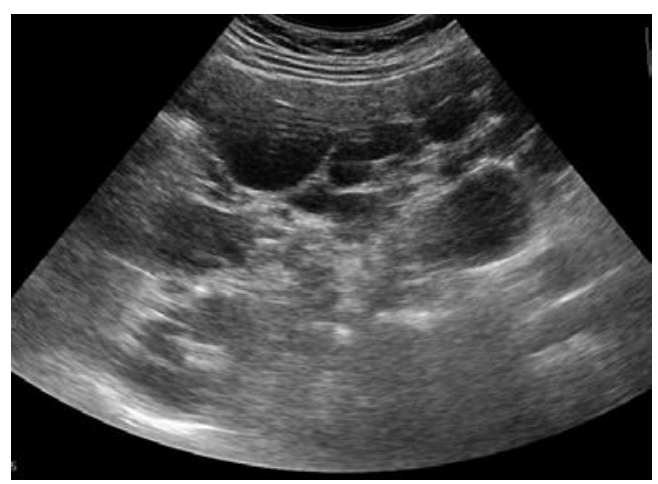

Fig 1. Ultrasonography revealed neumerous varying size cystic lesion without solid component .

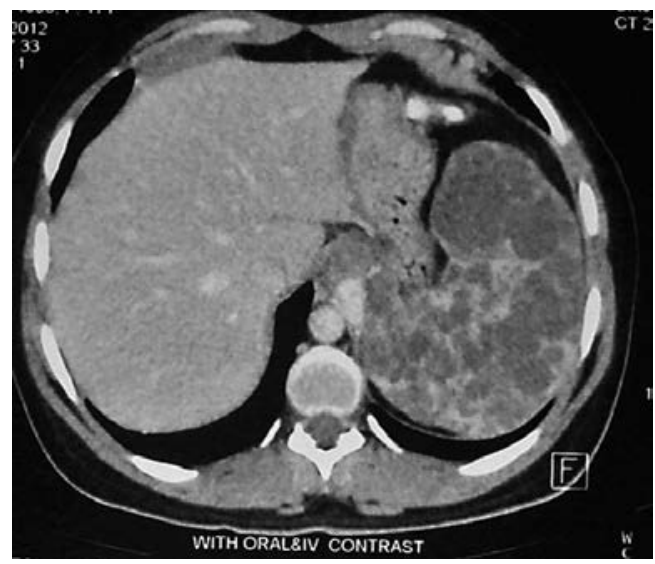

Fig 2. Contrast enhanced abdominal CT showing enlarged spleen with cystic area lesions

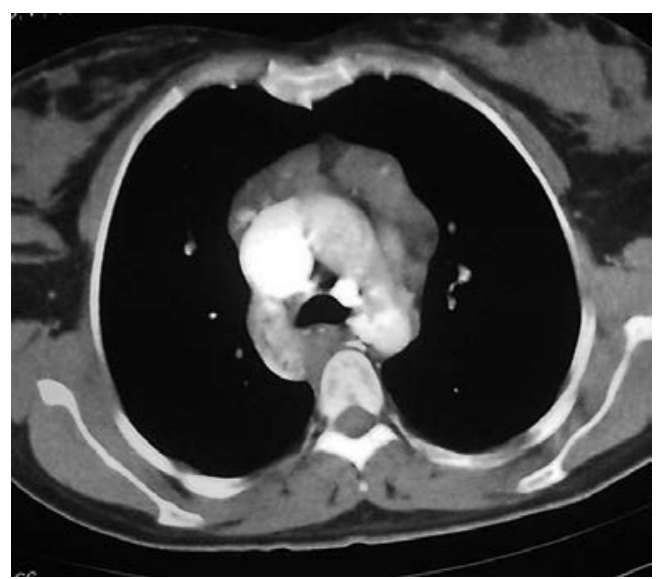

Fig 3. Contrast enhanced thoracic CT showing a lobulated hypodense mass encasing the great vessels without calcification. 


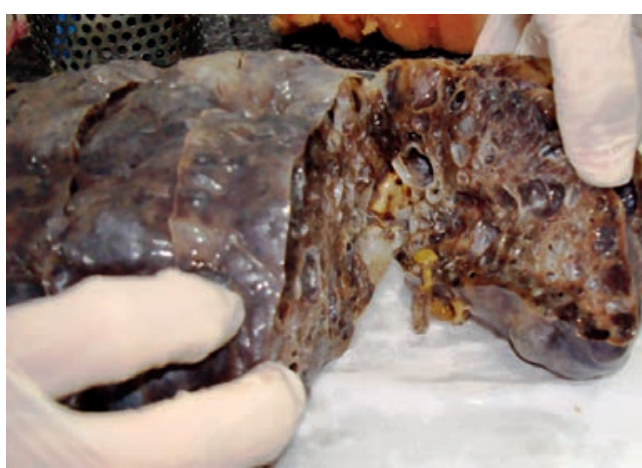

Fig 4. Enlarged spleen showed the nodular surface and cystic area lesions

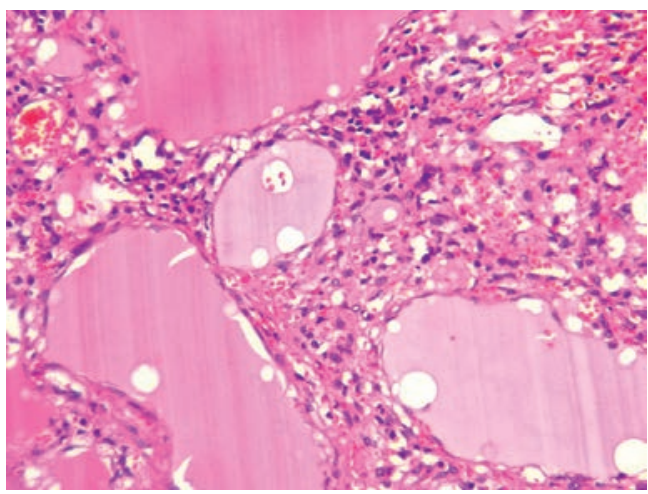

Fig 5. Histopathological aspect- variable size cystic space, walled with a flattened endothelial cells in a fibrovascular stroma

case of lymphangioma involving mesentery and retroperitoneum in a symptomatic patient [9]. The particularity of our case is the involvement of the spleen and the mediastinum in an adult as a asymptomatic condition.

Aspiration, drainage and sclerosis are some of the conservative managements that are accompanied with a high risk of recurrence. Whenever possible, the complete surgical resection should be performed. Some au- thors believe that if complete resection has a high risk of mortality or morbidity a partial resection should be considered [1]. Diathermy, radiotherapy and percutaneous sclerotherapy are the other therapeutic protocol that has been tried $[1,10]$.

\section{References:}

1. Surlin V, Georgescu E, Dumitrescu C, Râmboiu S, Comănescu MV, Ghiluşi M. Retropancreatic cystic lymphangioma - considerations upon a case. Rom J Morphol Embryol 2011; 52(1 Suppl): 493-496.

2. Castellón Pavón C, Lanchas Alfonso I, González Núñez MA, Amigo Lozano ML, del Amo Olea E. Splenic and adrenal lymphangiomatosis. Rev Esp Enferm Dig 2003; 95: 585-588, 581-584.

3. Xu GP, Shen HF, Yang LR, Ma Q, Gao BL. Splenic cystic lymphangioma in a young woman: case report and literature review. Acta Gastroenterol Belg 2011; 74: 334336.

4. Vezzoli M, Ottini E, Montagna M, et al. Lymphangioma of the spleen in an elderly patient. Haematologica 2000; 85: 314-317.

5. Luo CC, Huang CS, Chao HC, Chu SM, Hsueh C. Intraabdominal cystic lymphangiomas in infancy and childhood. Chang Gung Med J 2004; 27: 509-514.

6. Tsukamoto T, Tanaka S, Yamamoto T, et al. Laparoscopic excision of a retroperitoneal cystic lymphangioma: report of a case. Surg Today 2003; 33: 142-144.

7. De Perrot M, Rostan O, Morel P, Le coultre C. Abdominal lymphangioma in adults and children. Br J Surg 1998; 85: 395-397.

8. Chung SH, Park YS, Jo YJ, et al. Asymptomatic lymphangioma involving the spleen and retroperitoneum in adults. World J Gastroenterol 2009; 15: 5620-5623.

9. Kim DH, Byun JN, Jang JY. Cystic lymphangioma involving the mesentery and the retroperitoneum: A case report. J Korean Radiol Soc 2005; 52: 347-350.

10. Shankar KR, Roche CJ, Carty HM, Turnock RR. Cystic retroperitoneal lymphangioma: treatment by image-guided percutaneous catheter drainage and sclerotherapy. Eur Radiol 2001; 11: 1021-1023. 